\title{
UM MODELO DE QUATRO QUADRANTES PARA A DETERMINAÇÃO DA OFERTA E DA DEMANDA DE INTERNAÇÕES HOSPITALARES*
}

Alexandre Marinho $§$

\section{RESUMO}

Este trabalho apresenta um modelo geral para representar a oferta e a demanda de internações em hospitais, e avaliar os resultados da interação entre estas variáveis, e os seus determinantes, sobre a taxa de mortalidade hospitalar. Os exercícios desenvolvidos estão focalizados em variáveis disponíveis no Sistema Único de Saúde - SUS, com as quais são realizadas análises de estática comparativa, mas é aplicável a hospitais em geral.

Palavras-chave: internações hospitalares, oferta e demanda, taxas de mortalidade hospitalar, Sistema Único de Saúde - SUS.

\section{ABSTRACT}

In this paper a particular model is developed and used to explain the outcome of hospital mortality rate and quantity of inpatient care in hospital care markets. The model focuses on the Brazilian National Health System (Sistema Único de Saúde - SUS) but it may be helpful in portraying a broad range of health systems. The effects of movements in underlying factors that may cause shifts in both demand and supply are readily predictable.

Key words: inpatient care; supply and demand, hospital mortality rates, Brazilian National Health System - SUS.

JEL classification: I11, I12, I18.

O autor agradece os valiosos comentários de um parecerista anônimo. Todos o erros remanescentes são de minha inteira responsabilidade.

$\S$ Do Instituto de Pesquisa Econômica Aplicada - IPEA, da Universidade do Estado do Rio de Janeiro - UERJ, e bolsista do CNPq. E-mail: amarinho@ipea.gov.br.

Recebido em setembro de 2004. Aceito para publicação em julho de 2005. 


\section{INTRODUÇÃO}

\section{I.1 Apresentação}

O presente trabalho apresenta um modelo analítico geral para representar a oferta e a demanda de internação em hospitais, bem como as eventuais situações de equilíbrio entre estas duas dimensões do mercado de serviços hospitalares. O modelo é essencialmente teórico, mas acolhe, e valoriza, os achados disponíveis na literatura de caráter mais empírico. A motivação básica é a necessidade de tornar acessível, para economistas não especializados em Economia da Saúde, as complexas relações existentes entre as diversas variáveis que determinam a oferta e a demanda por internações hospitalares. Adicionalmente, a metodologia apresentada é útil para não-economistas, notadamente para os gestores de sistemas de saúde e de hospitais, que poderão apreender, de modo mais expedito, as possibilidades oferecidas pela aplicação do instrumental econômico no estudo do mercado hospitalar. O modelo está focalizado em variáveis disponíveis no Sistema Único de Saúde - SUS, na página do Departamento de Informação e Informática do SUS - DATASUS (wwww.datasus.gov.br), mas, com algumas adaptações, poderia ser aplicado em hospitais em geral.

\section{AS VARIÁVEIS RELEVANTES MAIS COMUNS EM ESTUDOS ECONÔMICOS SOBRE HOSPITAIS}

\section{II.1 Apresentação}

Os modelos de caráter econômico para representar o funcionamento de hospitais apresentam, usualmente, um conjunto mínimo de variáveis representativas dos elementos básicos observáveis no funcionamento destas instituições. Entre estes elementos destacam-se: a quantidade e a qualidade dos serviços prestados, a taxa de mortalidade, o prazo médio de permanência (internação) do conjunto dos casos tratados, e os custos operacionais. Além destas variáveis, também é freqüente a utilização de variáveis especificamente representativas da demanda, e que os hospitais não controlam diretamente, entre as quais se destacam: a gravidade dos casos, a idade, e a renda dos pacientes. É comum que a gravidade dos casos considere as conseqüências da própria idade dos pacientes. Também é usual que os pacientes sejam agrupados por níveis de risco. Nos Estados Unidos, em grande parte dos estudos, e inclusive para pagamentos governamentais pelas compras de serviços hospitalares, os diversos grupos de risco costumam ser agrupados nos diagnosis-related groups (DRGs). O leitor interessado nesta e em outras variáveis nem tão recorrentes tem diversas fontes de consulta, dependendo do contexto a ser enfatizado. Os dois trabalhos seminais enfocando os hospitais como unidades econômicas produtivas são o de Newhouse (1970) e o de Feldstein (1971). Harris (1977) enfatiza a dicotomia interna aos hospitais, onde os médicos demandam insumos aos administradores, ao mesmo tempo que ofertam serviços aos pacientes. Barnum e Kutzin (1993) avaliam a produção e os custos nos hospitais existentes em países em desenvolvimento. Em Butler (1995) são encontrados exaustivos estudos relacionados aos custos hospitalares. Santerre e Neun (2000) apresentam uma visão de Economia Industrial do setor hospitalar, com ênfase nos Estados Unidos. Sloan (2000) discute a implicação da existência de motivações lucrativas e não-lucrativas na atividade dos hospitais. Marinho e Façanha (2000) apresentam um resumo das variáveis úteis em estudos sobre a eficiência de hospitais. 


\section{II.2 A quantidade de serviços}

A primeira variável importante é um indicador da quantidade de atendimento. Este indicador pode ser, por exemplo, o número de internações ou de procedimentos específicos, o nível de complexidade ofertada para o paciente (mix de procedimentos e nível do aporte tecnológico), a quantidade (número total de procedimentos) e intensidade (número de procedimentos por unidade de tempo) de tratamento dispensado. Cada um destes elementos compõe a cesta de serviços que o hospital é capaz de produzir. Uma variável sintética, a “cesta de serviços” ofertada, poderá representar a quantidade média de cuidados ofertados para o paciente. Esta variável-síntese pode ser calculada como um índice ponderado de quantidades, que é função crescente de variáveis relacionadas com a urgência, a complexidade, a quantidade total, e a intensidade dos cuidados ofertados.

\section{II.3 A qualidade dos serviços}

A segunda variável que costuma ser explicitamente representada na atividade de hospitais é a qualidade do atendimento ofertado. Qualidade é uma medida complexa e controversa em saúde. McGlynn (1997) alerta que pacientes, prestadores de serviços e pagadores definem qualidade de modos diferentes. Pode-se ainda pensar, como usual, em qualidade de processos ou de produtos. $\mathrm{O}$ conceito de qualidade de processos e de produtos aplica-se tanto às práticas médicas quanto aos serviços de hotelaria prestados pelo hospital. Quantidades de exames, taxas de mortalidade, taxas de infecção hospitalar, tempo de espera em filas, e número de enfermeiros, entre outras, são todas variáveis que, isoladamente, ou em diferentes combinações, têm sido utilizadas para a representação da qualidade do atendimento hospitalar. No presente estudo, por conveniência analítica, será feita a opção de considerar, em separado, a qualidade representativa do nível de atendimento e de apoio, ou seja, da "hotelaria" e da "hospedagem" oferecida aos pacientes. Este conceito de qualidade é, propositadamente, mais limitado do que o conceito usual na literatura. Mas ajuda a contornar o difícil problema de separação (tanto no nível empírico como no nível conceitual) entre a qualidade dos serviços médicos prestados e a qualidade percebida pelos pacientes (ver, para mais detalhes, Enthoven e Vorhaus, 1997 e McGlynn, op. cit.). Outras dimensões da qualidade, incluindo as relacionadas com a qualidade técnica dos serviços médicos ofertados, estarão representadas na cesta de serviços médicos ofertada, que será uma medida ponderada dos diferentes esforços (considerando as suas diferentes quantidades e estágios tecnológicos) que a mão-de-obra e a tecnologia médica do hospital fazem para tratar do paciente. Butler (op. cit., especialmente no cap. 3) explicita as vantagens desta separação sobre a análise dos custos, mas alerta que a construção dessa média ponderada demanda um esquema classificatório, um sistema de pesos e uma fórmula agregadora, sendo que esta pode, ou não, ser linear. ${ }^{1}$

\section{II.4 As taxas de mortalidade}

A taxa de mortalidade hospitalar é obtida com a divisão do número de óbitos pelo número de casos tratados em um determinado período. Trata-se de um indicador que deve ser observado com muita cautela, pois não explicita as peculiaridades da população residente na área atendida pelos hospitais, nem o perfil da oferta de serviços, nem a gravidade dos casos tratados, nem o nível de complexidade dos procedimentos e diagnósticos realizados, e nem mesmo o papel do hospital nos sistemas de saúde. No caso do SUS, e de outros sistemas de saúde complexos, o sistema de referên-

1 Uma formalização possível da medida seria a cesta de serviços $Q=\sum w_{i} q_{i}, i=1 \ldots$ n, onde $w_{i}$ seria o peso do esforço do tipo $i$ e $q_{i}$ a respectiva quantidade desse esforço. 
cia e de contra-referência para os hospitais também exerce influência sobre os níveis e as distribuições das taxas de mortalidade entre os hospitais. Tais indicadores também não explicitam os problemas gerados por diferenças em tamanhos de amostras, freqüências de procedimentos, aleatoriedades etc. Diferentes alternativas de medidas (razões de mortalidade, taxas padronizadas, taxas específicas etc.) poderiam fornecer resultados diferentes. O leitor interessado nestes aspectos deve consultar, entre outros, Rouquayrol e Almeida Filho (1999) para mais detalhes.

\section{II.5 O prazo médio de permanência}

O prazo médio de permanência, ou prazo médio de internação, mede o tempo médio que um paciente passa internado nos hospitais. Trata-se de um indicador de desempenho bastante polêmico, sem que haja um consenso conceitual a respeito das interpretações e do uso que se possa fazer dele. Uma primeira vertente de interpretações admite que o prazo médio de internação é um indicador de qualidade. Neste sentido, quanto menor o prazo, ceteris paribus, melhor para o paciente, e melhor a qualidade do atendimento ou do hospital. Embora exista uma tendência declinante dos prazos de internação no mundo, alguns cuidados devem ser observados, pois sempre se pode argüir que altas precoces impliquem pacientes em pior situação de saúde (“quicker but sicker”). Implícita nesta argumentação está a hipótese de endogeneidade do indicador. Os hospitais, em algum grau, controlariam os prazos de internação. Mas determinar se esse controle ocorreria à custa da saúde dos pacientes é uma questão empírica, fora do alcance do presente texto. De qualquer modo, existe a possibilidade de que menores prazos decorram dos avanços da Medicina, ou de cuidados diários mais intensivos. Abstraindo-se as questões de saúde, e entrando na seara da eficiência, essa combinação pode, ou não, resultar em custos totais mais elevados. Uma outra interpretação indica que os prazos são exógenos, diretamente relacionados com a complexidade, ou gravidade dos casos. Nesta hipótese, maiores prazos em uma unidade podem estar relacionados com fatores fora do controle do hospital, e não indicam má qualidade de atendimento, vis-à-vis aos prazos menores em outras unidades de saúde.

Uma vertente alternativa, e complementar à anterior, interpreta o prazo médio de internação como uma medida de produção. Maior número de dias de internação implica maiores níveis de produção. Neste caso, devem ser observadas as influências que modos alternativos de remuneração dos hospitais exercem sobre os prazos de permanência. Orçamentos fixos e por procedimentos (caso da maioria dos hospitais no SUS), e pagamentos per capita, tenderiam a incentivar menores prazos médios de internação nos hospitais. Pagamentos per diem tenderiam a tornar maiores os prazos de permanência. Verifica-se, em todo o mundo, uma queda dos prazos médios de permanência.

Dadas as complexidades dessa variável, serão adotadas, no presente estudo, duas hipóteses básicas muito pouco restritivas, e que contemplam as duas linhas argumentação anteriores: ceteris paribus, a cesta de serviços médicos prestados aumenta com o prazo de permanência, e também, ceteris paribus, quanto maior a qualidade (no sentido da hotelaria) dos serviços, menor o prazo de permanência. Melhorias na qualidade técnica dos serviços médicos reduzem os prazos de permanência e deslocarão para baixo a curva de oferta, conforme será explicitado mais adiante no texto, especialmente na seção III.2.

\section{II.6 Os custos em hospitais}

Seguindo uma tendência existente na literatura (ver Barnum e Kutzin, op. cit.), o modelo do presente trabalho adota uma função de custos totais (ao invés de custos médios) de curto prazo, supondo que o estoque de capital não seja facilmente modificado em menos de um ano. Ademais, di- 
versos autores (entre os quais Scuffham et alii, 1996; e Marinho e Façanha, op. cit.) apontam para a ausência de evidências de que hospitais estejam em equilíbrio de longo prazo. No modelo aqui apresentado os custos de produção, gerados por uma tecnologia exógena, e com preços de fatores também exógenos, são separáveis, no sentido de que o hospital poderia ser separado em duas unidades, uma produzindo quantidade e outra qualidade, com o custo de cada output podendo ser perfeitamente alocado ao respectivo processo produtivo. Uma das dimensões essenciais da qualidade do atendimento hospitalar é a chamada "hotelaria" ou "hospedagem" ou apoio e atendimento para os pacientes, cuja tecnologia é bastante independente e "separável" da tecnologia médica (diagnósticos e tratamentos). No presente trabalho, a dimensão de qualidade estará acarretando estes custos (hotel costs). Dito de outro modo, não existiria produção conjunta e nem custos em comum (common costs). Assim, o custo marginal de produção de cada um dos outputs é independente do nível de produção do outro output (para mais detalhes, ver Butler, op. cit.). Os custos diários de hospedagem dos pacientes (lavanderia, limpeza etc.) tendem a ser constantes para um mesmo paciente, mas podem variar bastante entre tratamentos distintos. Já os custos do tratamento (diagnósticos, medicamentos e procedimentos diversos) costumam ser mais elevados na admissão e na alta dos pacientes, não sendo lineares ao longo do tempo, nos casos de internação. Os custos fixos referem-se, usualmente, ao estoque de capital fixo (leitos, instalações e equipamentos diversos) hospitalar e à mão-de-obra empregada ou referencia o tamanho dos hospitais, medido principalmente pelo número de leitos ativos. O custo total é dado pela soma dos custos de produção de cada bem separadamente. Como a derivada cruzada dos custos em relação aos diferentes produtos é nula, não existem custos em comum e nem economias ou deseconomias de escopo, ou seja, variações nos níveis de quantidade não implicam variações nos níveis de qualidade de serviços prestados e viceversa (para mais detalhes, ver Butler, op. cit.). Giuffrida et alii (2000) encontram evidências da presença de economias de escala e da ausência de economias de escopo na administração de serviços de saúde (National Health System - NHS) na Inglaterra entre 1989 e 1995.

$\mathrm{Na}$ realidade, em hospitais, mesmo a distinção entre insumos e produtos não é trivial. Inúmeros bens intermediários (throughputs) são gerados no processo de atendimento hospitalar. É muito comum a adoção desses bens intermediários (dias de internação, exames, consultas, cirurgias etc.), ora como bens finais, ora como insumos, dependendo das conveniências do problema tratado, por absoluta impossibilidade de se obter uma solução definitiva para tal problemática, em termos conceituais ou empíricos. O bem final, a saúde, é de difícil mensuração.

\section{II.7 A função-objetivo e o nível geral de produção nos hospitais: planejadores $\mathrm{x}$ sistema de preços}

Na hipótese mais comum na microeconomia, os preços (incluindo os custos) de mercado seriam o mecanismo de alocação de recursos nos hospitais, determinando o nível de produção ao fazer os preços iguais aos custos marginais. Mas se em um hospital sem fins lucrativos o planejador social obtém utilidade marginal positiva na solução do problema de maximização, ou seja, se o planejador "gosta" da produção de algum dos bens, o hospital não lucrativo produz em um ponto no qual os custos marginais são maiores que os preços. Sob essa hipótese, o hospital não lucrativo produz mais do bem (preferido) que o lucrativo. Esta hipótese merece consideração em sociedades com escassez de oferta de serviços hospitalares. O oposto ocorre se o planejador não gosta da produção. Neste caso, o hospital não lucrativo produz menos do que o lucrativo. É interessante notar que é recorrente, na literatura, a hipótese de que gestores hospitalares, que são usualmente médicos, gostam da produção, ou seja, têm predileção por grandes organizações (para mais detalhes, ver Neun e Santerre, op. cit.; e Sloan, op. cit.). Este comportamento teria origem em várias motivações. Entre as mais freqüentemente apontadas estão as razões de prestígio dos médicos (que cresceria 
com o tamanho e abrangência da clínica), a necessidade de atender a uma demanda incerta, a incorreta percepção de custos, problemas de agency (os médicos induziriam a demanda) e, mesmo, a procura por sinecuras.

Resta um ponto bastante importante a ser assinalado. Em termos estritamente econômicos, sabe-se que uma firma não será socialmente eficiente, e estará produzindo bens em excesso, se estiver operando em um ponto no qual o custo marginal de cada bem é maior do que o preço do bem respectivo, caso os preços sejam considerados boas representações para o valor social dos bens. Maiores níveis de produção não são um valor absoluto em Economia, onde os conceitos de otimização e de eficiência também são valorizados. Não existe, em Economia da Saúde, consenso a respeito da aplicabilidade direta e nem da razoabilidade de tais conceitos na análise de hospitais. De qualquer modo, não parece razoável afirmar que a administração dos preços dos serviços hospitalares possa estar totalmente desvinculada dos custos. Definir se a decisão de produção deve ser deixada a cargo dos planejadores ou do sistema dos preços ultrapassa o alcance do presente texto.

\section{UM MODELO DE QUATRO QUADRANTES PARA A REPRESENTAÇÃO DA OFERTA E DA DEMANDA POR INTERNAÇÃO EM HOSPITAIS}

\section{III.1 Apresentação}

Na presente seção será incorporada uma análise do impacto da produção de serviços hospitalares sobre a taxa de mortalidade hospitalar esperada (ou efetiva) para cada paciente, em um modelo de quatro quadrantes, com variáveis observadas e registradas no SUS e na maioria dos sistemas hospitalares. A taxa de mortalidade é um indicador clássico, razoavelmente simples e rotineiro, de resultados (outcomes) originados pela oferta de serviços em hospitais no Brasil (eg. Brasil, 1991; Yazlle Rocha et alii, 1997; Travassos et alii, 1999; Schramm e Szwarcwald, 2000; Martins et alii, 2001; Silva, 2003; e Marinho, 2003) entre outros, e no exterior (eg. Green et alii, 1990; Park et alii, 1990; Green et alii, 1991; D’Hoore et alii, 1993; Romano, 1993; McClellan et alii, 1994; Zuckerman et alii, 1994; Iezzoni et alii, 1997; Iezzoni et alii, 1998; Gowrisankaran e Town, 1999; Jarman, 1999; Selim et alii, 2002), entre outros. Para o presente trabalho, este indicador apresenta a vantagem de poder ser facilmente incorporado ao lado da demanda do modelo, pois afeta diretamente o nível de bem-estar dos pacientes quando eles consomem os serviços ofertados pelos hospitais. Com estas características, o modelo simplificado que será apresentado guarda similaridades metodológicas com os modelos de oferta e demanda usuais em Economia. ${ }^{2}$

No lado da oferta de serviços, entre as variáveis importantes, que são insumos produtivos (inputs) básicos ou intermediários (throughputs) na determinação da taxa de mortalidade hospitalar, pode-se arrolar: a qualidade (no sentido limitado do nível de hotelaria e de serviços gerais de atendimento e de apoio) e o tempo de duração da internação. Além destes insumos mais gerais, na oferta, temos insumos mais estreitamente relacionados com a ação e a tecnologia médica: complexidade (mix de procedimentos e nível do aporte tecnológico) e a quantidade (número total de procedimentos por caso) e intensidade (número de procedimentos por caso por unidade de tempo) ou o dual destas variáveis, como os custos. Em nosso modelo, a variável sintética "cesta de serviços médicos” representará a quantidade média de cuidados médicos oferecidos para o paciente. A cesta de serviços médicos poderia ser calculada (embora isto não seja feito no presente estudo) como um índice ponderado de quantidades, que seria função crescente de variáveis relacionadas

2 Particularmente, o modelo guarda similaridades com os modelos IS-LM (Hicks-Hansen) da teoria macroeconômica. 
com a urgência, a complexidade, a quantidade total e a intensidade dos cuidados médicos ofertados. A cesta de serviços médicos é, portanto, uma medida do esforço que a mão-de-obra e a tecnologia médica do hospital faz para tratar do paciente.

No lado da demanda, pode-se, por conveniência analítica, vislumbrar duas categorias de variáveis: primeiramente, as características mais específicas do campo da saúde como a urgência, a complexidade, a quantidade, e a intensidade do tratamento demandado pelo caso (a cesta de serviços médicos). Portanto, na demanda, esta cesta de serviços representará a quantidade média de cuidados médicos demandados pelo caso. Como na oferta, essa média poderia ser calculada (o que também não será feito neste trabalho) como um índice ponderado de quantidades, que seria função crescente de variáveis relacionadas com a urgência, a complexidade, a quantidade e a intensidade de cuidados médicos demandados. Estas características devem ser acompanhadas de informações mais gerais sobre os pacientes, tais como: a idade, e as condições de vida (ou, na ausência desta, a renda permanente) dos pacientes. Conforme já foi dito, especificar a qualidade na demanda é uma tarefa complexa e, além da própria conceituação ser problemática, avaliar o grau de percepção e as possibilidades de contratação (verifiability) da mesma também não são tarefas triviais. Por razões metodológicas, adota-se a hipótese simplificadora, bastante aceitável, de que a qualidade demandada cresce com a renda, fazendo, deste modo, que a qualidade (da hotelaria e dos serviços gerais) também seja incorporada na demanda, e que a cesta de serviços médicos seja dimensionalmente idêntica na oferta e na demanda. A probabilidade de morte (ou a taxa de mortalidade esperada ou efetiva) imputável ao caso é o resultado final (outcome) relevante na demanda. Os custos do tratamento também poderiam ser, de modo análogo ao caso da oferta, uma medida da cesta de serviços médicos demandada.

O modelo simplificado de quatro quadrantes para representar a demanda e para a oferta de internações, apresentado a seguir, determina o lócus de equilíbrio de variáveis fundamentais na atuação de hospitais. Com hipóteses bastante aceitáveis e usuais na literatura, consegue-se determinar os impactos de cada uma dessas variáveis sobre o equilíbrio no modelo. Como é usual em Economia, pode-se passar, sem restrições adicionais, da demanda individual para a demanda agregada e da oferta individual para a oferta agregada. Diversos exercícios de estática comparativa podem ser realizados.

\section{III.2 A oferta de serviços hospitalares}

Para caracterização da oferta de serviços são formuladas as seguintes hipóteses:

1. as administrações dos hospitais escolhem a qualidade de serviços e o tempo de internação de cada caso, pois podem regular o fluxo de serviços que oferecem para cada paciente;

2. o nível de complexidade, a quantidade de procedimentos e a intensidade do tratamento ofertado são os componentes da cesta de serviços médicos ofertada para cada caso, que é uma variável endógena aos hospitais;

3. como resultado da escolha da qualidade do tratamento e do prazo de internação, os hospitais ofertam combinações de taxas de mortalidade e de níveis de cesta de serviços médicos;

4. o prazo de internação (incluindo reinternações) é uma função crescente da cesta de serviços médicos ofertada;

5. o prazo de internação (incluindo reinternações) é função decrescente da qualidade dos serviços ofertados;

6. a taxa de mortalidade esperada (ou efetiva) é função decrescente da qualidade dos serviços ofertados. 
As hipóteses 1-6 são todas amplamente utilizadas e discutidas na literatura, por exemplo, em Arrow (1963), em Barnun e Kutzin (op. cit.), em Butler (op. cit.) e em Harris (op. cit.). Supondo que todas as funções sejam diferenciáveis, são obtidas as relações, descritas a seguir, que determinam a oferta de serviços de internação no hospital. A numeração das equações segue os quadrantes do gráfico onde cada uma delas será representada. A variável q é a cesta de serviços médicos ofertada. Assim, q é um índice de quantidades que mede a complexidade, a quantidade e a intensidade do tratamento ofertado, sendo crescente com todas estas variáveis. Sejam as equações a seguir:

$\left(I V^{s}\right) . T=f(q), \partial f(q) / \partial q \geq 0$, onde $T$ é o prazo de internação e;

$\left(\mathrm{III}^{\mathrm{s}}\right) . \mathrm{T}=\mathrm{g}(\mathrm{Q}), \partial \mathrm{g}(\mathrm{Q}) / \partial \mathrm{Q} \leq 0$, onde $\mathrm{Q}$ é a qualidade dos serviços prestados e $\mathrm{g}(\mathrm{Q})$ é inversível. Conclui-se que: $\mathrm{Q}=\mathrm{g}^{-1}(\mathrm{~T})=\mathrm{h}(\mathrm{T}), \partial \mathrm{h}(\mathrm{T}) / \partial \mathrm{T} \leq 0\left(\mathrm{III}^{\mathrm{s} 1}\right)$;

$\left(\mathrm{II}^{\mathrm{s}}\right) . \mathrm{M}^{\mathrm{s}}=\mathrm{j}(\mathrm{Q}), \partial \mathrm{j}(\mathrm{Q}) / \partial \mathrm{Q} \leq 0$, onde $\mathrm{M}^{\mathrm{s}}$ é a taxa de mortalidade hospitalar esperada ou efetiva.

A simples manipulação das equações anteriores permite obter a função composta:

$\left(I^{s}\right) \cdot M^{s}=j(h(f(q)))=m(q), \partial m(q) / \partial q \geq 0$.

Na oferta, a mortalidade hospitalar é diretamente proporcional à complexidade do caso. Podese pensar que, em equilíbrio, quanto maior a taxa de mortalidade esperada, maior a cesta de serviços médicos ofertada pelo hospital. A Figura 1, a seguir, apresenta um diagrama de quatro quadrantes representando a oferta de serviços hospitalares de internação, de acordo com as equações $\mathrm{I}^{\mathrm{s}}-\mathrm{IV}^{\mathrm{s}}$, representadas nos quadrantes respectivos.

Figura 1 - Diagrama de quatro quadrantes para a oferta de internações hospitalares

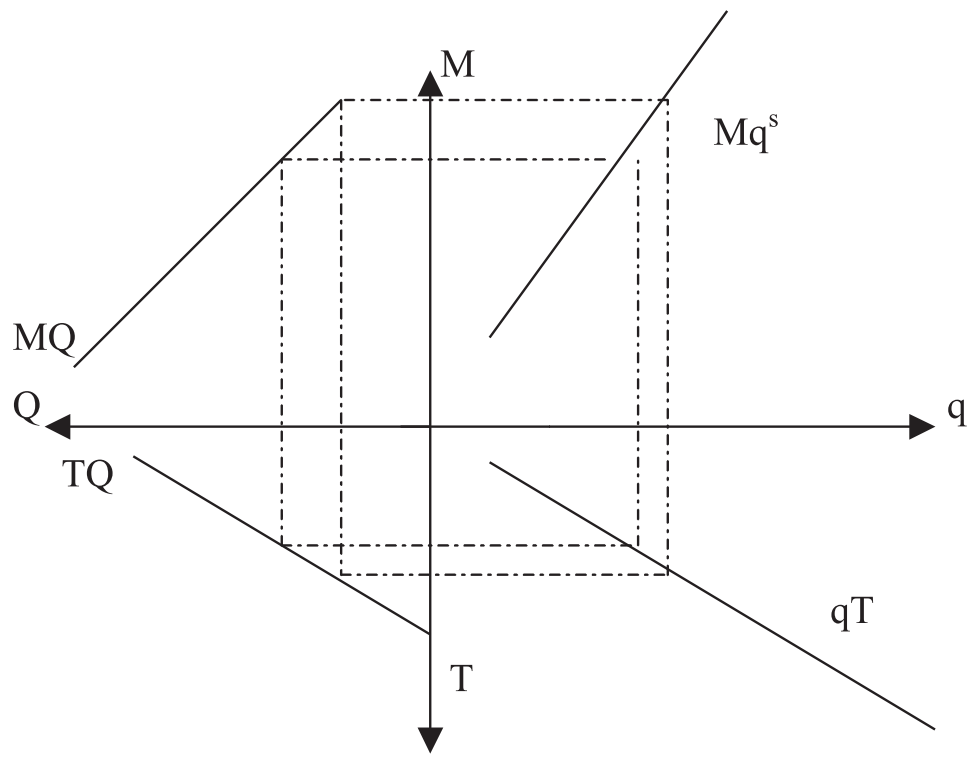

O diagrama, conforme será visto, permite realizar, com facilidade, diversos exercícios de estática comparativa. A Figura 2, a seguir, ilustra a situação em que os prazos médios de internação são reduzidos para todos os níveis de qualidade. 
Figura 2 - Redução no prazo médio de internação com qualidade constante

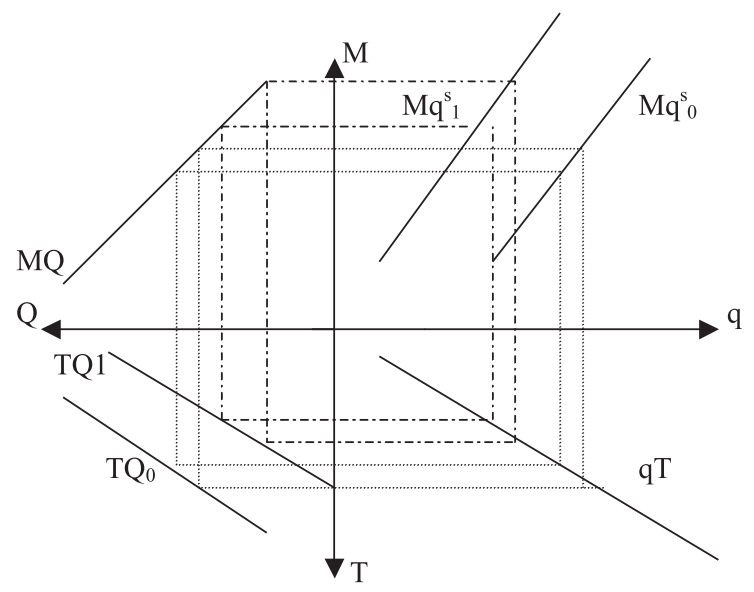

Redução dos prazos de internação com qualidade constante: suponha-se, na Figura 2, que a curva que relaciona o prazo de internação e a qualidade se desloca de $\mathrm{TQ}_{0}$ para $T Q_{1}$. Neste caso, para o mesmo nível de qualidade, o tempo ofertado de internação diminui. Como conseqüência, a curva que relaciona complexidade e mortalidade se desloca de $\mathrm{Mq}_{0}^{\mathrm{s}}$ para $\mathrm{Mq}_{1}^{\mathrm{s}}$. Para cada nível de complexidade deve-se esperar maior taxa de mortalidade. É o caso conhecido como "quicker but sicker". Ceteris paribus, um esquema de incentivos que provoque redução nos prazos de internação, sem contrapartida de aumentos na qualidade dos serviços, pode ocasionar tais resultados. Os pagamentos fixos por procedimentos, adotados no SUS, estão, em princípio, nesta categoria de incentivos. Diversos exercícios similares podem ser realizados com as demais curvas do gráfico.

Prazo de internação fixo (curva de oferta quebrada): outro exercício de interesse é supor que o prazo de internação tenha um limite superior fixado independentemente da qualidade ofertada. Algumas empresas de saúde suplementar tentam implementar esta política. Neste caso, a oferta de serviços também adquire um limite superior a partir do qual as taxas de mortalidade cresceriam abruptamente. Este resultado está apresentado na Figura 3, a seguir.

Figura 3 - Curva de oferta "quebrada": internações com prazo máximo limitado independentemente da qualidade

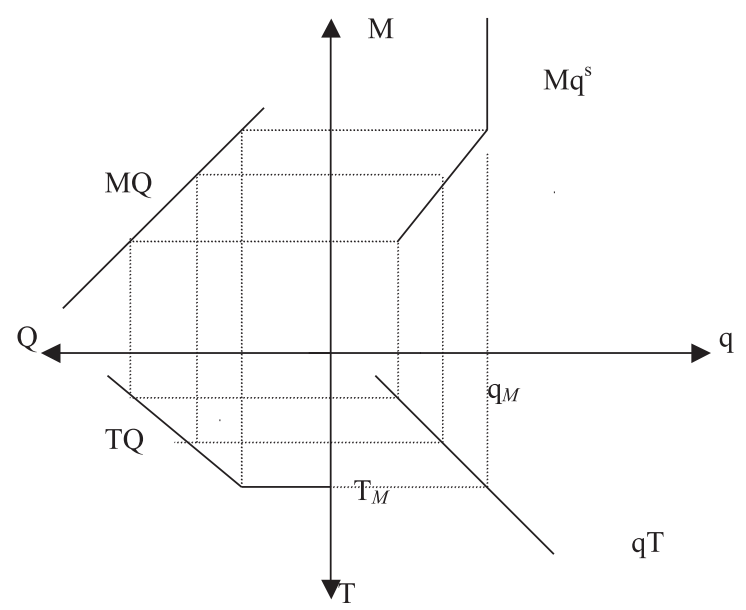


A curva de oferta "quebrada" tem um segmento perfeitamente inelástico. Se o prazo máximo de internação permitido é fixado em $\mathrm{T}_{M}$, o hospital não oferta serviços a partir de $\mathrm{q}_{M}$.

Os avanços da medicina reduzem os prazos de internação: uma situação também relevante simula um eventual avanço da medicina, que torna os prazos de internação menores para todos (ou alguns) os níveis de cesta de serviços médicos ofertada. Neste caso, a curva qT se desloca de $\mathrm{qT}_{0}$ para $\mathrm{qT}_{1}$, e a curva de oferta se desloca de $\mathrm{Mq}_{0}^{\mathrm{s}}$ para $\mathrm{Mq}_{1}^{\mathrm{s}}$, o que implica menores taxas de mortalidade para todos os níveis de tratamento, conforme a Figura 4, a seguir. Se as práticas médicas se deteriorassem ocorreria o movimento oposto, com aumento na taxa de mortalidade.

Figura 4 - Avanços da medicina reduzem os prazos de internação em diversos níveis de cesta de serviços médicos ofertada

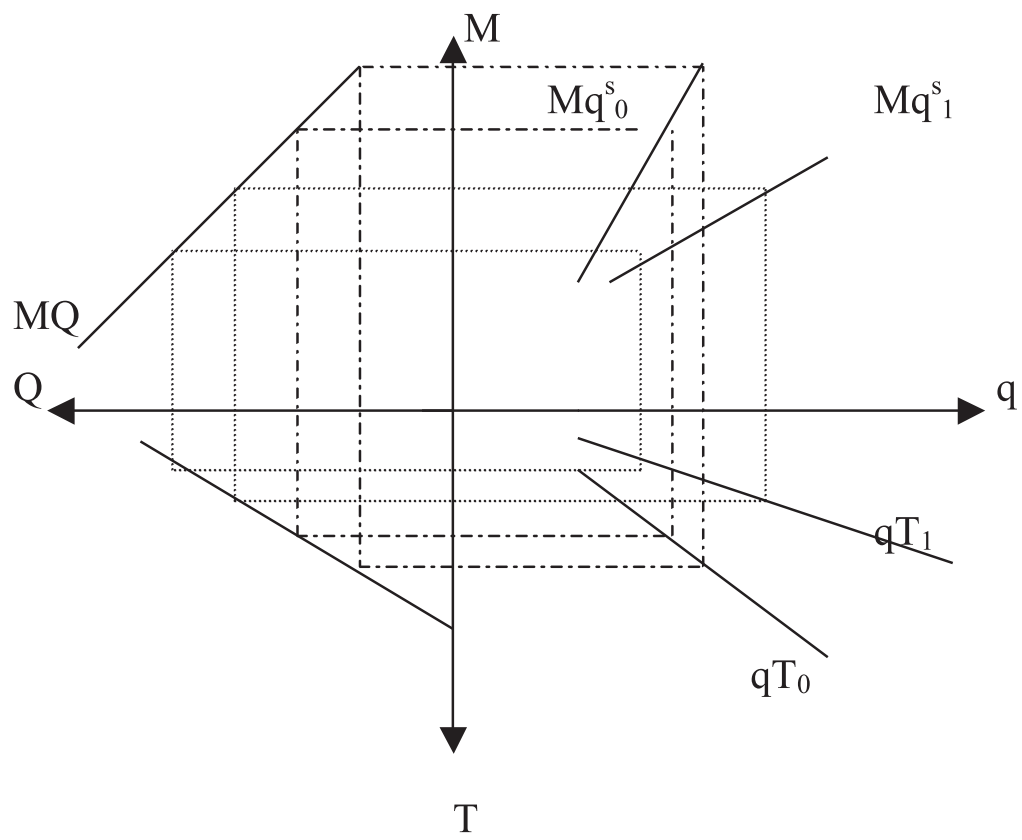

\section{III.3 A demanda por serviços hospitalares}

$\mathrm{Na}$ demanda de serviços são formuladas as seguintes hipóteses:

1. o nível de complexidade, a quantidade de procedimentos e a intensidade do tratamento demandado são os componentes da cesta de serviços médicos demandada em cada caso, que é escolhida pelos pacientes, de acordo com os médicos (que são agentes dos pacientes) sendo, portanto, variável endógena na demanda;

1. a cesta de serviços médicos demandada em cada caso é função crescente da idade dos pacientes;

1. a renda permanente do paciente (ou de suas famílias) é função crescente da idade dos pacientes;

1. a taxa de mortalidade esperada (ou efetiva) é função decrescente da renda (ou das condições de vida) dos pacientes.

Todas as hipóteses anteriores encontram respaldo na literatura. A hipótese 1 está em Arrow (op. cit.), Barnun e Kutzin (op. cit.), em Butler (op. cit.), e em Harris (op. cit.). As hipóteses 2 e 4 encontram-se, entre outros, em Barnun e Kutzin (op. cit.), em Butler (op. cit.), em Harris (op. cit.) 
e em Grossman (1972). A hipótese 3 está, por exemplo, em Becker (1975) e em Grossman (op.cit.). Dadas as hipóteses 1-4, e supondo que todas as funções sejam diferenciáveis, são obtidas as seguintes relações, determinantes da demanda por serviços de internação no hospital. A numeração das equações segue os quadrantes do gráfico onde cada uma delas será representada:

$\left(I^{d}\right) . q=u(I), \partial u(I) / \partial I \geq 0$, onde I é a idade do paciente e $u(I)$ é inversível.

Logo, $\mathrm{I}=\mathrm{u}^{-1}(\mathrm{q})=\mathrm{v}(\mathrm{q}), \partial \mathrm{v}(\mathrm{q}) / \partial \mathrm{q} \geq 0\left(\mathrm{IV}^{\mathrm{d} 1}\right) ;$

$\left(\mathrm{III}^{\mathrm{d}}\right) . \mathrm{R}=\mathrm{w}(\mathrm{I}), \partial \mathrm{w}(\mathrm{I}) / \partial \mathrm{I} \geq 0$, onde $\mathrm{R}$ é a renda permanente do paciente;

$\left(\mathrm{II}^{\mathrm{d}}\right) . \mathrm{M}^{\mathrm{d}}=\mathrm{z}(\mathrm{R}), \partial \mathrm{z}(\mathrm{R}) / \partial \mathrm{R} \leq 0$, onde $\mathrm{M}_{\mathrm{d}}$ é a taxa de mortalidade esperada ou efetiva.

Manipulando-se as fórmulas obtém-se:

$\left(I^{d}\right) \cdot M^{d}=z(w(v(q)))=n(q), \partial n(q) / \partial q \leq 0$.

Na demanda, a cesta de serviços médicos demandada varia de modo inverso com a taxa de mortalidade. O paciente vai desejar consumir maiores quantidades de serviços que proporcionem menores taxas de mortalidade. Pode-se chegar a tal conclusão com um raciocínio similar ao utilizado para outros bens em Economia. Se a taxa de mortalidade no tratamento hospitalar esperada ao "consumir" tratamento é alta, ao invés de tratamento o paciente pode demandar mais outros bens, como, por exemplo, atenção religiosa, ou lazer. Em um caso limite, se a morte é certa, nenhum tratamento é demandado. Por outro lado, se a cura é certa, a demanda por internação é muito elevada. Ao escolher entre hospitais, os pacientes demandam mais os hospitais com menores taxas de mortalidade em casos similares. Também é possível realizar exercícios de estática comparativa na demanda. A Figura 5, a seguir, apresenta um diagrama de quatro quadrantes para demanda por serviços de internação de acordo com as equações $\mathrm{I}^{\mathrm{d}}-\mathrm{IV}^{\mathrm{d}}$, representadas nos quadrantes respectivos.

Figura 5 - Diagrama de quatro quadrantes para a demanda por internações hospitalares

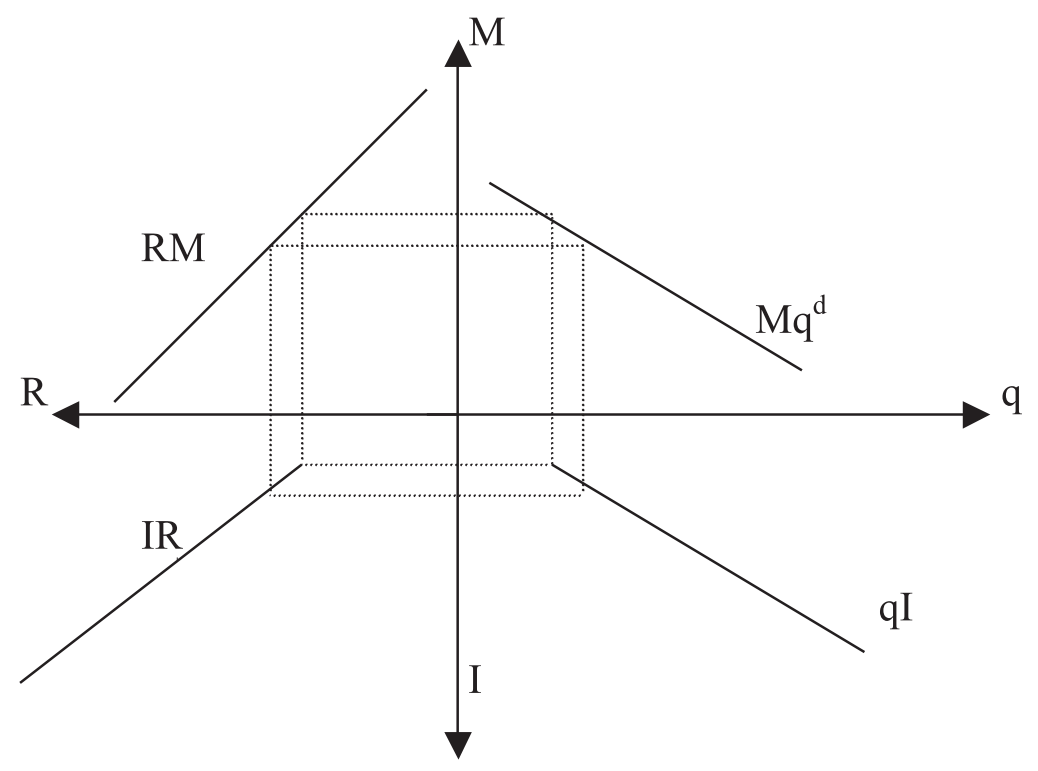


Exercícios de estática comparativa também são possíveis na demanda. A Figura 6, a seguir, retrata a situação em que a renda permanente média diminui em todos os níveis de idade.

Figura 6 - A renda permanente média diminui em todos os níveis de idade

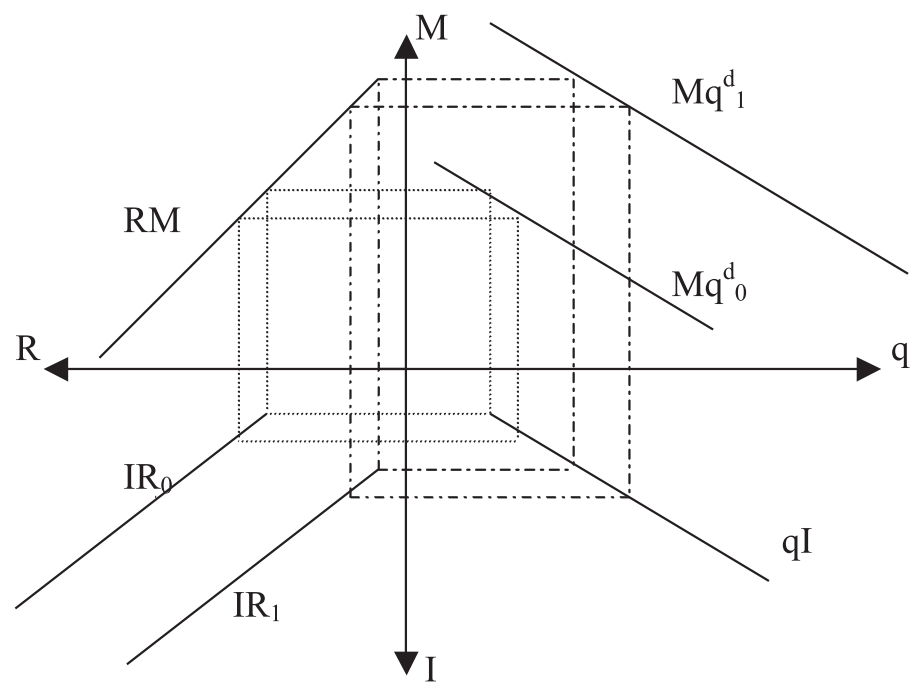

Queda da renda média dos pacientes: suponha-se que a renda permanente média dos pacientes diminua em todas as faixas etárias. Neste caso, na Figura 6 a curva que relaciona idade e renda se desloca de $\mathrm{IR}_{0}$ para $\mathrm{IR}_{1}$. Conseqüentemente, a curva de demanda $\mathrm{Mq}_{0}^{\mathrm{d}}$ se desloca para $\mathrm{Mq}_{1}^{\mathrm{d}}$ e deve-se esperar um aumento da taxa de mortalidade para todos os níveis de tratamento demandados. Outros exercícios podem ser executados no modelo, mas deve-se atentar que, em circunstâncias normais, o hospital não controla, diretamente, nenhuma das variáveis da demanda. Uma variável importante e fora do controle dos hospitais é a renda dos pacientes, conforme analisado na Figura 7, a seguir.

Figura 7 - Curva de demanda de internações com renda fixa

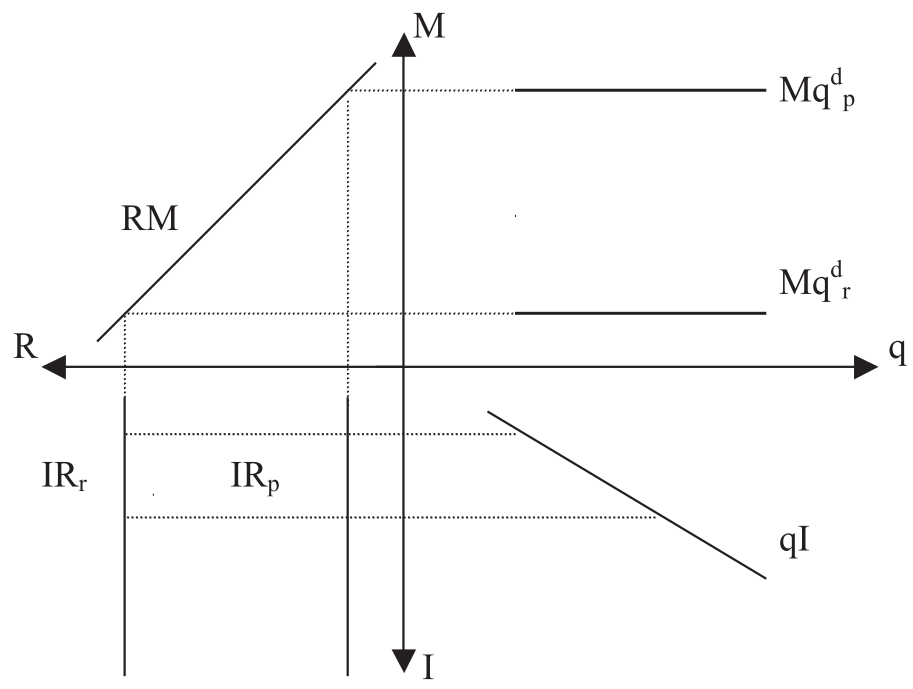


A renda permanente média dos pacientes não varia com a idade: suponha-se, como na Figura 7, que a renda média dos pacientes seja fixa ao longo da idade. Neste caso, a renda será $\mathrm{R}={ }_{\mathrm{w}}(\mathrm{I})=\mathrm{K}$ (constante). A taxa de mortalidade é $\mathrm{M}^{\mathrm{d}}={ }_{\mathrm{Z}}(\mathrm{R})=\mathrm{z}(\mathrm{w}(\mathrm{I})=\mathrm{K})=\mathrm{n}(\mathrm{K})$, também constante. Vê-se que a taxa de mortalidade não depende da cesta de serviços médicos, e a demanda se torna perfeitamente elástica. Esta hipótese poderia ser observada, por exemplo, em sociedades extremamente pobres, com a pobreza prevalecendo em todas as gerações, conforme representado pela renda fixa $\mathrm{IR}_{\mathrm{p}}$, que origina a demanda $\mathrm{Mq}_{\mathrm{p}}^{\mathrm{d}}$. A situação social oposta, com elevada riqueza, também estaria assim representada pela renda fixa $\mathrm{IR}_{\mathrm{r}}$, que origina a demanda $\mathrm{Mq}_{\mathrm{r}}^{\mathrm{d}}$, com taxa de mortalidade inferior à observada na sociedade pobre.

É interessante notar que, neste caso, avanços da medicina, deslocadores da oferta, são incapazes de reduzir as taxas de mortalidade nos hospitais. Mas são ainda capazes de, às mesmas taxas de mortalidade, tratar de casos mais graves.

\section{III.4 Equilíbrio entre a oferta e a demanda por internações}

Das equações $\left(\mathrm{I}^{\mathrm{s}}\right)$ e $\left(\mathrm{I}^{\mathrm{d}}\right)$ obtém-se a relação de equilíbrio entre a oferta e a demanda por serviços nos hospitais:

$\mathrm{M}^{\mathrm{s}}=\mathrm{m}(\mathrm{q}), \partial \mathrm{m}(\mathrm{q}) / \partial \mathrm{C} \geq 0$

$\mathrm{M}^{\mathrm{d}}=\mathrm{n}(\mathrm{q}), \partial \mathrm{n}(\mathrm{R}) / \partial \mathrm{R} \leq 0$.

Em equilíbrio, $\mathrm{M}^{\mathrm{s}}=\mathrm{M}^{\mathrm{d}}=\mathrm{M}^{*}$.

O modelo determina, na interseção da oferta e da demanda, a taxa de mortalidade de equilíbrio $\mathrm{M}^{*}$, caso ela exista, dado o nível de cesta de serviços médicos de equilíbrio $\mathrm{q}^{*}$. Este ponto será exatamente o ponto $\mathrm{E}\left(\mathrm{q}^{*}, \mathrm{M}^{*}\right)$ na Figura 8 , a seguir.

Figura 8 - Equilíbrio entre a oferta e a demanda por internações

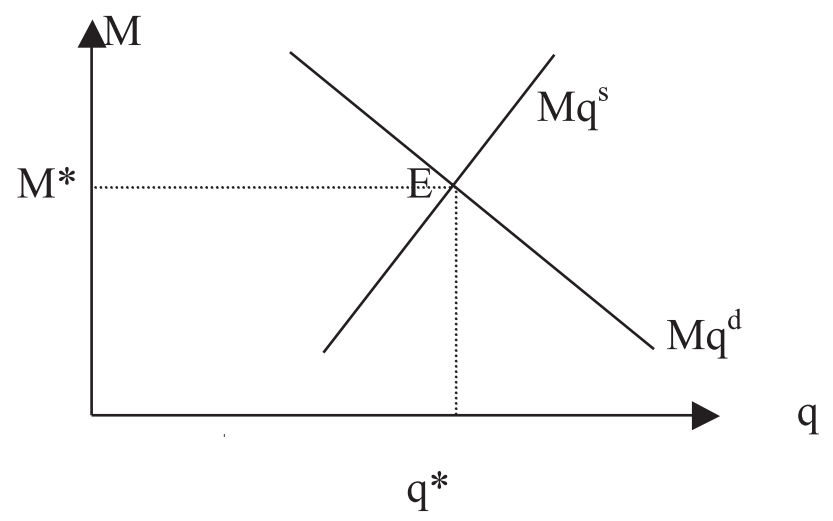

\section{III.5 Comentários sobre a aplicabilidade do modelo}

Dois aspectos são fundamentais na aplicabilidade do modelo para a análise de taxas de mortalidade em hospitais. O primeiro refere-se à necessidade de se analisar com rigor as variáveis exógenas determinantes da demanda e da oferta de internações. Os resultados obtidos pelos hospitais dependem de variáveis que estas instituições não controlam. Em seguida, é imperativo que se ava- 
liem todas as variáveis do modelo, em conjunto com os incentivos dados aos hospitais, e com as características dos mesmos. Por exemplo, as motivações, os incentivos, e as características presentes em hospitais lucrativos e não lucrativos não são inteiramente coincidentes no Sistema Único de Saúde - SUS e em outros sistemas de saúde.

Exercícios de agregação também são realizáveis, permitindo conclusões sobre subconjuntos relevantes de um sistema hospitalar. Note-se que se, por exemplo, na Figura 9, a seguir, a curva de oferta $\mathrm{Mq}^{\mathrm{s} 0}$ se desloca para $\mathrm{Mq}^{\text {s1 }}$ à esquerda, ocorrerá uma elevação da taxa de mortalidade de equilíbrio para algum valor $\mathrm{M}^{* *}$, acima de $\mathrm{M}^{*}$ e uma redução dos serviços prestados de $\mathrm{q}^{*}$ para $\mathrm{q}^{* *}$. Este movimento poderia ser resultado da transformação de um hospital não lucrativo em entidade que vise lucros, com a conseqüente redução da oferta de serviços. É muito importante ressaltar, entretanto, que uma análise de equilíbrio geral talvez revele que o resultado economicamente ótimo, no caso do deslocamento da oferta para a esquerda, seja exatamente uma maior taxa de mortalidade hospitalar. Este resultado ocorrerá se o sistema de preços, incluídos os preços dos insumos (os custos), for um bom guia para alocação de recursos em saúde. A expansão da oferta hospitalar para além do ponto ótimo poderia estar significando deslocamento de recursos de outros setores, até mesmo de outros subsetores da saúde, que estariam aquém do ponto ótimo, piorando o nível de bem-estar geral da sociedade. A expansão da oferta de internação hospitalar poderia se dar à custa, por exemplo, de programas de prevenção ou de proteção à saúde. $\mathrm{O}$ modelo faz apenas uma análise de equilíbrio parcial simplificada de internações em hospitais, não contemplando todos os programas, subsetores e mercados em saúde.

\section{Figura 9 - A curva de oferta se desloca para a esquerda}

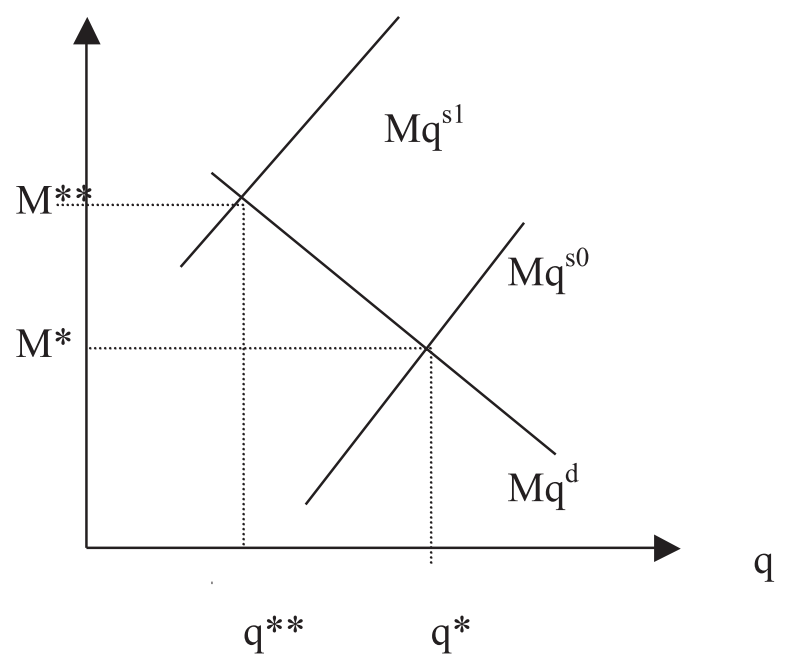

Um outro exercício possível representa o equilíbrio com baixa taxa de mortalidade, reduzida quantidade de serviços prestados, alto prazo de permanência e baixa qualidade. Um exemplo clássico com este perfil, em passado recente, no Brasil, é o da Clínica Psiquiátrica, onde os pacientes simplesmente moravam em hospitais, durante anos, com oferta de tratamento praticamente nula. A Clínica Psiquiátrica envolve os diversos transtornos e síndromes mentais, neuróticos, de personalidade e comportamentais. Inclui, entre outros distúrbios, os devidos ao uso de substâncias psicoativas ou retardos mentais. Frank e McGuire (2000) apontam que, usualmente, estes transtornos são crônicos, podem surgir em pessoas jovens, e que envolvem a perda de capacidade de decisão dos indivíduos. Esses autores ressaltam que grande parte dos esforços na Clínica Psiquiátrica destina- 
se a tratar dos sintomas, não curando os pacientes em prazos muito curtos. Como, além destes pontos, existe um estigma associado com os riscos que tais doentes trazem para "segurança pública", muitas vezes, inclusive em países desenvolvidos, o tratamento psiquiátrico é meramente um instrumento de controle e de exclusão social. Conseqüentemente, nesta especialidade são comuns os elevados prazos de internação e a baixa intensidade dos tratamentos, mesmo em casos muito complexos. Em 2002, o prazo médio de permanência das internações classificadas no capítulo V (Transtornos Mentais e Comportamentais) da Classificação Estatística Internacional de Doenças e Problemas Relacionados à Saúde - Décima Revisão/CID X é de 60,6 dias, bem acima da média geral, de apenas 6,2 dias. Note-se que em Clínica Psiquiátrica a média de permanência geral no SUS foi de 63,8 dias. O custo diário de internação, no mesmo capítulo $\mathrm{V}$, seria de $\mathrm{R} \$ 26,03$, ou seja, um valor bem abaixo da média geral do SUS, que foi de $\mathrm{R} \$ 74,35$. O custo total médio (por caso) da internação neste capítulo da CID X foi $\mathrm{R} \$ 1.576,90$, ou seja, bem superior à média geral, que ficou em $\mathrm{R} \$ 461,54$. Trata-se, portanto, de uma internação de longo prazo, que tem custo diário baixo e custo total elevado. Chalkley e Malcomson (2000) observam que na Psiquiatria o prazo de permanência é uma estatística suficiente para os custos. Hospitais deste tipo estão adaptados a baixos níveis de complexidade, reduzida quantidade de serviços prestados por caso e elevados prazos de permanência. Nestes casos, pode-se observar a existência de taxa de mortalidade baixa, independentemente do comportamento da oferta, em associação com baixo nível de qualidade, conforme é ilustrado no gráfico da Figura 10, a seguir. Note-se que, no caso em tela, um deslocamento moderado da curva de demanda $\mathrm{Mq}^{\mathrm{d} 0}$ para a curva $\mathrm{Mq}^{\mathrm{d} 1}$ à direita, causada por alguma epidemia repentina (de dengue, por exemplo), elevaria, dramaticamente, a taxa de mortalidade de equilíbrio, pois tal configuração hospitalar não é adequada para trabalhar com elevada demanda de serviços (q elevado).

Figura 10 - Equilíbrio compatível com baixa taxa de mortalidade, reduzida quantidade de serviços prestados, alto prazo de permanência e baixa qualidade

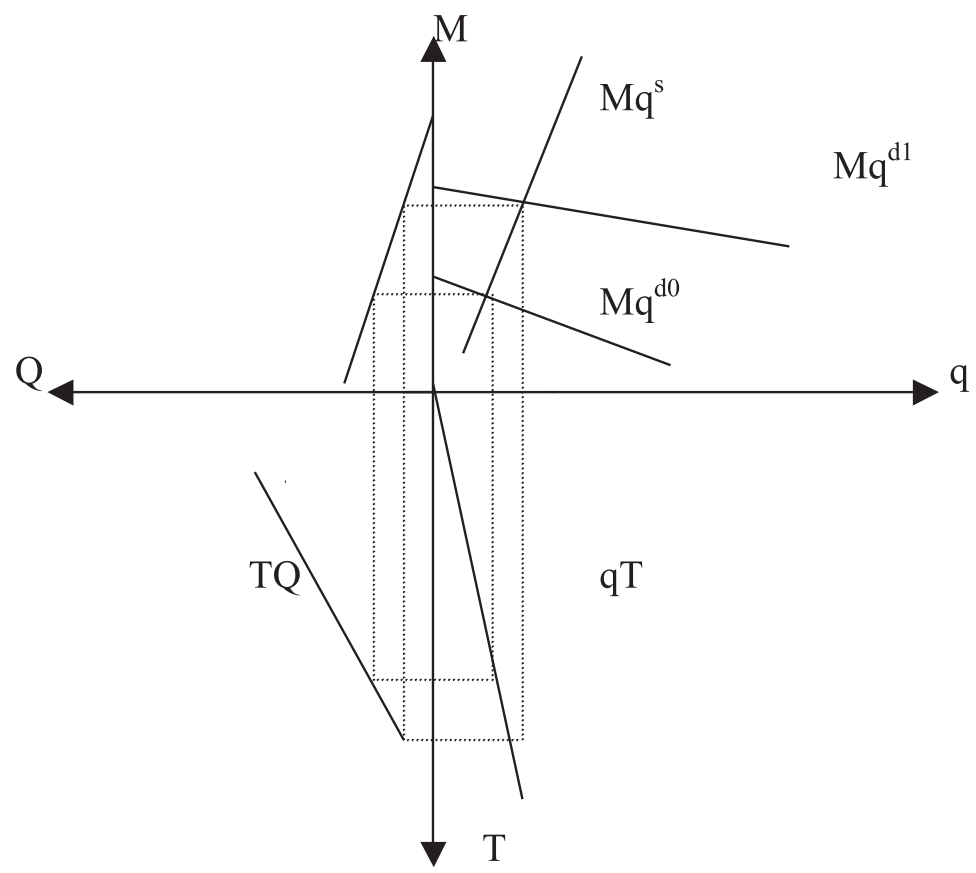

Observação: para não sobrecarregar a figura, as curvas qI, IR e RM, todas elas determinantes da demanda, não foram desenhadas. 


\section{III.6 Introduzindo custos no modelo}

O problema dos custos: a complexidade dos casos tratados é um dos principais componentes da cesta de serviços médicos efetivamente prestados em cada caso. Trata-se de uma variável decisiva na determinação da oferta, da demanda e do provável valor de equilíbrio da taxa de mortalidade dos hospitais. Entretanto, a definição conceitual e a representação empírica da complexidade dos casos tratados em hospitais não são tarefas triviais. Menos restritiva é a suposição de que as correlações entre complexidade, cestas de serviços médicos e custos devem ser diferentes de zero, e supostamente positivas. Em termos empíricos, a adoção dos custos pode ser vantajosa. Custos (ou, ao menos, as despesas) podem ser mais facilmente definidos e medidos do que a complexidade e do que a cesta de serviços médicos. Em termos empíricos, os custos admitem uma unidade de medida indiscutível, a moeda. Com algumas precauções, poderíamos substituir a cesta de serviços médicos pelos custos totais. Na oferta, os custos totais devem ser função crescente do prazo de internação e da magnitude da cesta de serviços médicos (ver Butler op. cit, especialmente a seção 3.3). A curva "cesta de serviços médicos x tempo de internação" (qT) poderia, então, ser substituída por uma curva "custos totais x tempo de internação" (CT), também com inclinação positiva. Na demanda, pode-se supor que os custos totais sejam função crescente da idade, ao menos a partir da idade adulta. Neste caso, a curva "cesta de serviços médicos x idade" (qI), que tem inclinação positiva, poderia ser substituída por uma curva "custos totais x idade" (CI), também com inclinação positiva. O espaço "custos totais x taxa de mortalidade" se apresentaria conforme a Figura 11, a seguir.

Os esforços implícitos na cesta de serviços refletem a complexidade dos tratamentos. Não está disponível, no DATASUS, nenhuma contrapartida empírica para a complexidade dos tratamentos, desagregada por hospitais. Mas uma razoável representação empírica dessa variável existe para as diferentes Unidades da Federação. A enorme gama de procedimentos de alta complexidade realizados no SUS está disponível na página do DATASUS na internet. E a alta complexidade representa o estado-da-arte disponível e aplicado no SUS. Está apresentado, no Apêndice deste texto, um resultado econométrico exploratório, e limitado, com o objetivo de determinar a correlação entre uma proxy de alta complexidade e os custos totais, em termos de AIH, dos hospitais do SUS, agregados por Unidades da Federação, no ano de 2002.

\section{Figura 11 - Modelo relacionando taxa de mortalidade e custos totais}

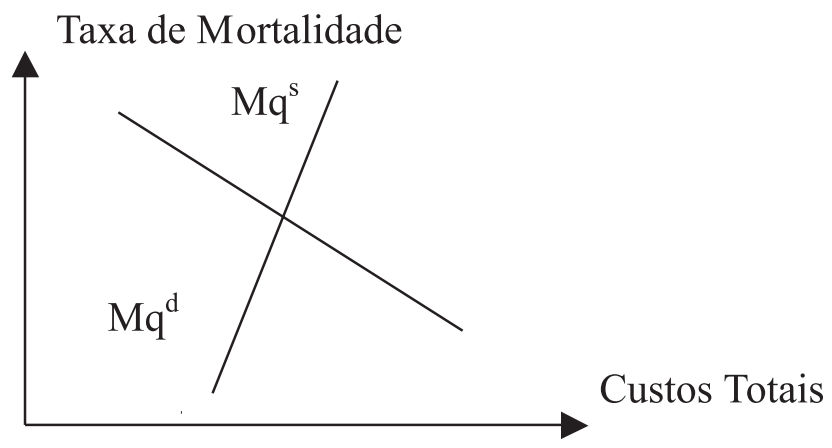

No gráfico "custos totais x taxa de mortalidade" vê-se que, na oferta, dada a inclinação positiva da curva $\mathrm{Mq}^{\mathrm{s}}$, casos com maiores probabilidades (taxas) de mortalidade custam mais caro. $\mathrm{Na}$ demanda, dada a inclinação negativa da curva $\mathrm{Mq}^{\mathrm{d}}$, os pacientes estão dispostos a pagar mais por menores probabilidades (taxas) de mortalidade. 
Se a oferta for pouco elástica, ou seja, se os hospitais relutarem em tratar casos com custos elevados, fica-se na situação em que deslocamentos da demanda para a direita, causados, por exemplo, por envelhecimento, ou empobrecimento da população, elevam drasticamente as taxas de mortalidade de equilíbrio. Esta é uma hipótese que não pode ser descartada para hospitais lucrativos. Novamente cabe ressaltar que em uma análise de equilíbrio geral este novo equilíbrio, com maiores taxas de mortalidade, seria o ótimo social se preços e custos forem indicadores decisivos na alocação de recursos em saúde.

\section{CONSIDERAÇÕES FINAIS}

É possível relacionar as taxas de mortalidade aos níveis de serviço prestados e aos custos hospitalares em um modelo simples de oferta e de demanda de serviços de internação. Este modelo é aplicável ao Sistema Único de Saúde - SUS, e permite a realização de diversos modelos de estática comparativa. $\mathrm{O}$ atendimento de picos de demanda, em hospitais com oferta inelástica, pode gerar elevações muito grandes das taxas de mortalidade. Se a opção for o atendimento em hospitais com oferta elástica, podem ocorrer grandes custos ou ineficiências econômicas. Este dilema, eficiência econômica versus redução da mortalidade hospitalar, aparentemente não tem solução fora do consenso e das limitações do sistema democrático. A Economia permitiria apenas, neste problema específico, avaliar quantos anos de vida adicionais uma unidade monetária poderia comprar em cada um dos segmentos do sistema hospitalar ou fora dele (no Programa de saúde da Família - PSF, por exemplo). Realizar esta avaliação de forma cuidadosa é uma tarefa para trabalhos posteriores.

\section{APÊNDICE: INVESTIGANDO A CORRELAÇÃO ENTRE CUSTOS E A CESTA DE SERVIÇOS MÉDICOS}

No modelo testado, a variável dependente é o custo, em termos de Autorização de Internações Hospitalares - AIH, dos procedimentos de alta complexidade realizados nos hospitais agregados por Unidades da Federação. Neste caso, a variável independente é cesta de serviços médicos, definida como o total dos procedimentos de alta complexidade. Os dados referem-se a 27 Unidades da Federação em 2002. Como estão sendo utilizados dados em cross-section, foi adotada a recomendação expressa em Madalla (2001) de aplicação de logaritmos nas variáveis visando minimizar os efeitos da presença de heteroscedasticidade no modelo. Esta recomendação é reforçada pelo fato de que não se pode garantir a priori a linearidade da relação entre os valores brutos das variáveis. Um modelo linear também foi testado, mas não será exibido, pois os resultados são muito semelhantes. Deve-se ressaltar que a produção real é maior do que os valores do DATASUS, pois existe represamento do pagamento das AIHs por parte do Governo Federal.

Tabela 1A - Regressão nas Unidades da Federação. Variável dependente: logaritmo do custo da alta complexidade em AIH

\begin{tabular}{lccccc}
\hline Variável Independente & Coeficiente (B) & Desvio Padrão (D.P.) & B/(D.P.) & P[Z/z] & Média da Variável \\
\hline Constante & 2,591 & 0,122 & 21,237 & 0,000 & \\
Log. Cesta de serviços médicos & 1,185 & 0,033 & 35,909 & 0,000 & 6,8260 \\
\hline
\end{tabular}

$\mathrm{R}^{2}=0,981$. 
Estatísticas do teste de White com 2 graus de liberdade: $\chi^{2}=2,68$, e $p=0,2621$, rejeita-se a presença de heteroscedasticidade.

Pode-se verificar, na Tabela $1 \mathrm{~A}$, que o modelo não rejeita, ao nível de $1 \%$, a existência de uma correlação positiva entre o logaritmo dos custos em AIH de alta complexidade e o logaritmo da cesta de serviços médicos nas Unidades da Federação em 2002. Resta dizer que seriam recomendáveis a apuração e a divulgação no DATASUS, dos valores despendidos por itens de custo, em cada um dos hospitais dos SUS.

Dados os resultados da regressão anterior, vê-se que além de teoricamente consistente, seria também empiricamente possível, no modelo de quatro quadrantes, substituir a variável complexidade pela variável representativa dos custos em AIH. Ressalte-se, novamente, que este é apenas um exercício empírico para o modelo no SUS. Em termos teóricos, como visto, necessita-se apenas de uma função de custos conceitualmente bem elaborada e que seja crescente com a cesta de serviços médicos.

\section{REFERÊNCIAS BIBLIOGRÁFICAS}

Arrow, K. Uncertainty and the welfare economics of medical care. The American Economic Review, v. LIII, n. 5, p. 941-973, dec. 1963.

Barnum, H.; Kutzin, J. Public hospitals in developing countries: resource use, cost, financing. Baltimore, USA: International Bank for Reconstruction and Development/The World Bank, 1993.

Becker, G. Human capital; a theoretical and empirical analysis with special reference to education. $2^{\text {nd }}$ ed. NY, USA: Columbia University Press, 1975.

Brasil. Programa brasileiro da qualidade e produtividade. Critérios para a geração de indicadores da qualidade e produtividade no serviço público. Ministério da Economia, Fazenda e Planejamento, Instituto de Pesquisa Econômica Aplicada - IPEA, nov. 1991.

Butler, J. R. G. Hospital cost analysis. The Netherlands: Kluwer Academic Publishers, 1995.

Chalkley, M.; Malcomson, J. M. Governmental purchasing of health services. In: Culyer, A. J.; Newhouse, J. P. (eds.), Handbook of health economics. V. 1A. Amsterdam, The Netherlands: Elsevier-North-Holland, 2000, p. 847-887.

D'hoore, W.; Sicotte, C.; Tilquin, C. Risk adjustment in outcome assessment: the Charlson comorbidity index. Methods of Information in Medicine, 32, p. 382-387, 1993.

Enthoven, A. C.; Vorhaus, C. B. A vision of quality in health care delivery. Health Affairs, v. 16, n. 3, p. 44-57, May/June 1997.

Feldstein, M. S. Hospital cost inflation: a study of nonprofit price dynamics. The American Economic Review, v. LXI, n. 5, p. 853-872, dec. 1971.

Frank, R. G.; McGuire, R. G. Economics and mental health. In: Culyer, A. J.; Newhouse, J. P. (eds.), Handbook of health economics. V. 1B. Amsterdam, The Netherlands: Elsevier-North-Holland, 2000, p. 893-954.

Giuffrida, A.; Gravelle, H.; Sutton, M. Efficiency and administrative costs in primary care. Journal of Health Economics, v. 19, p. 983-1006, 2000.

Gowrisankaran, G.; Town, R. J. Estimating the quality of care in hospitals using instrumental variables. Journal of Health Economics 18, p. 747-767, 1999.

Green, J.; Wintfeld, N.; Passman, L. Analyzing hospital mortality. The consequences of diversity in patient mix. The Journal of the American Medical Association, v. 265, n. 14, p. 1849-1853, April 10, 1991. 
Green, J.; Wintfeld, N.; Sharkey, P.; Passman, L. The importance of severity of illness in assessing hospital mortality. The Journal of the American Medical Association, v. 263, n. 2, p. 241-246, Jan. 12, 1990.

Grossman, M. On the concept of health capital and the demand for health. Journal of Political Economy, v. 80, n. 2, p. 223-255, 1972.

Harris, J. The internal organization of hospitals: some economic implications. The Bell Journal of Economics, v. 8, n. 42, p. 467-482, autumn 1977.

Iezzoni, L. et alii. Differences in procedure use, in-hospital mortality, and illness severity by gender for acute myocardial infarction patients. Medical Care, v. 35, n. 2, p. 158-171, 1997.

Iezzoni, L. et alii. Predicting in-hospital deaths from coronary artery bypass graft surgery: do different severity measures give different predictions? Medical Care, v. 36, n. 1, p. 28-39, Jan. 1998.

Jarman, B. et alii. Explaining differences in english hospital death rates using routinely collected data. British Medical Journal, 318, p. 1515-1520, June 1999.

McGlynn, E. A. Six challenges in measuring the quality of health care. Health Affairs, v. 16, n. 3, p. 7 21, May/June 1997.

Maddala, G. S. Introduction to econometrics. $3^{\text {rd }}$ ed. N.Y., USA: John Wiley \& Sons, LTD, 2001.

Marinho, A. Avaliação da eficiência técnica nos serviços de saúde nos municípios do Estado do Rio de Janeiro. Revista Brasileira de Economia, v. 57, n. 3, jul./set. 2003.

Marinho, A.; Façanha, L. O. Hospitais universitários: avaliação comparativa de eficiência técnica. Economia Aplicada, v. 4, n. 2, p. 315-349, abr./jun. 2000.

Martins, M.; Travassos, C.; Noronha, J. C. Sistema de informações hospitalares como ajuste de risco em índices de desempenho. Revista de Saúde Pública, v. 35, n. 2, p. 185-192, 2001.

McClellan, M.; McNeil, B. J.; Newhouse, J. P. Does more intensive treatment of cause myocardial infarction in the elderly reduce mortality? The Journal of the American Medical Association, v. 272, n. 11, p. 859-866, Sep. 21, 1994.

Newhouse, J. P. Toward a theory of nonprofit institutions: an economic model of hospital. The American Economic Review, v. LX, n. 1, p. 64-74, March 1970.

Park, R. E. et alii. Explaining variation in hospital death rates. randomness, severity of illness, quality of care. The Journal of the American Medical Association, v. 264, n. 4, p. 489-489, July 25, 1990.

Romano, P. S. Can administrative data be used to compare the quality of health care? Medical Care Review, v. 50, n. 4, p. 451-477, Winter 1993.

Rouquayrol, M. Z.; Almeida Filho, N. Epidemiologia \& saúde. Rio de Janeiro, Brasil: MDSI, Editora Médica e Científica Ltda., 1999.

Santerre, E. R.; Neum, P. S. Health economics. Theories, insights, and industry studies. Revised Edition. Ohio, USA: South-Western, Thomson Learning, 2000.

Schramm, J. M. A.; Szwarwald, C. L. Sistema hospitalar como fonte de informações para estimar a mortalidade neonatal e a natimortalidade. Revista de Saúde Pública, v. 34, n. 3, p. 272-279, 2000.

Scuffham, P. A.; Devlin, N. J.; Jaforullah, M. The structure of costs and production in New Zealand public hospitals: an application of the transcendental logarithmic variable cost function. Applied Economics, 28, p. 75-85, 1996.

Selim et alii. Risk-adjusted mortality rates as a potential outcome indicator for outpatient quality assessments. Medical Care, v. 40, n. 3, p. 237-245, March 2002.

Silva, L. K. Validity of the risk adjustment approach to compare outcomes. Cadernos de Saúde Pública, v. 19, n. 1, p. 287-295, 2003. 
Sloan, F. Not-for-profit ownership and hospital behavior. In: Culyer, J.; Newhouse, J. P. A. (eds.), Handbook of health economics. V. 1B. Amsterdam, The Netherlands: Elsevier-North-Holland, 2000, p. 1141-1174.

Travassos, C.; Noronha, J. C.; Martins, M. Mortalidade hospitalar como indicador de qualidade: uma revisão. Ciência e Saúde Coletiva, v. 4, n. 2, p. 367-381,1999.

Yazlle Rocha, J. S.; Simões, B. J. G.; Guedes, G. L. M. Assistência hospitalar como indicador da desigualdade social. Revista de Saúde Pública, v. 31, n. 5, p. 479-487, out. 1997.

Zuckerman, S.; Hadley, J.; Iezzoni, L. Measuring hospital efficiency with frontier cost functions. Journal of Health Economics, 13, p. 255-280, 1994. 